\title{
Biochemical Effect of General Anesthesia on Women's Mood and Depression
}

\author{
Shadia A. Fathy ${ }^{1}$, Mohamed R. Mohamed ${ }^{1 *}$, Mohamed S. Shorbagy ${ }^{2}$, Mohamed A. Ahmed ${ }^{2}$ and Aya \\ Z. Salem ${ }^{1}$ \\ ${ }^{I}$ Department of Biochemistry, Faculty of Science, Ain Shams University, 11566 Abbassia, Cairo, Egypt, \\ ${ }^{2}$ Department of anesthesia and intensive care unit, Faculty of Medicine, Ain Shams University, 11566 Abbassia, Cairo, \\ Egypt
}

ARTICLE INFO

Article history:

Received 10 August 2015

Accepted 21 September 2015

Keywords:

General anesthesia;

depression;

estrogen;

progesterone;

dopamine;

serotonin.

\begin{abstract}
A B S T R A C T
The influence of general anesthesia on mood and depression is not fully understood. The purpose of this study was to investigate the effect of general anesthesia on main female sex hormones; estrogen and progesterone, and biogenic amine neuromediators; dopamine and serotonin (5-hydroxytryptamine, 5-HT). The selection of these hormones and neurotransmitters was based on their being mood stabilizers and natural antidepressant. The present results show significant elevation in progesterone and dopamine concomitant with significant reduction in estrogen level and a non-significant change in serotonin level of female patients, postoperatively, compared to the preoperative levels. These results indicate that general anesthesia doesn't induce negative mood or any sort of depression. Instead, it enhances mood via the increase in serum levels of dopamine and progesterone and alleviates the decreased synthesis in serotonin level, induced by the decrease in estrogen level, by reducing or inhibiting serotonin uptake, postoperatively. In addition, anesthetic agents help in great relieving of pain by increasing endogenous morphine, as a natural pain killer, through rising dopamine level.
\end{abstract}

\section{Introduction}

Anesthesia describes a complex state composed of immobility, amnesia, hypnosis (sleep or loss of consciousness), analgesia, and muscle relaxation [1] Anesthesia enables patients to tolerate surgical procedures that would otherwise inflict unbearable pain, potentiate extreme physiologic exacerbations and result in unpleasant memories ${ }^{[2]}$. A growing body of evidence suggests that depression and panic disorder are both associated with an increased frequency of clinical pain complaints ${ }^{[3]}$. It was believed that depression and mood disorder are caused by an imbalance of brain chemicals, namely neurotransmitters. Some of these chemicals, related to mood disorders, are serotonin, dopamine, and norepinephrine ${ }^{[4]}$.

General anesthesia has massive effects on neurotransmitter activity in the brain. It can change basal transmitter levels by interacting with neuronal activity, transmitter synthesis, release, reuptake and metabolism. By that way general anesthesia can affect great number of neurotransmitter systems and receptors ${ }^{[1]}$. It affects a

\footnotetext{
*Corresponding author.

E-mail address: mohamed_elsotohi@sci.asu.edu.eg
}

number of different acetylcholine and glutamate receptors in the brain, glycine receptors in the spinal cord and neurotransmitter receptors including the gammaaminobutyric acid (GABA) receptor. Furthermore, the serotonergic system may play a role during general anesthesia, where it was demonstrated that alterations in serotonergic neurotransmission in the hippocampus have been linked to depression and anxiety as well as to changes in arousal and cognition ${ }^{[5]}$.

Mood disorders specifically affect women than men and lead to change in her quality of life and her ability to fulfill her usual roles as workers, mothers and spouses. Females are specifically at greater risk for developing several chronic pain disorders and women exhibit greater sensitivity to noxious stimuli compared with men and this is interpreted as the consequence of a direct role of estrogens in modulation of pain perception and mood ${ }^{[6]}$. Female sex hormones, estrogen and progesterone play a role in modulating the beneficial and adverse effects of general anesthesia in women and this is likely to influence the rate and overall quality of recovery from general anesthesia ${ }^{[7]}$. 
Estrogen, progesterone and androgen receptors have been identified in mammalian brain and possess actions distinct from reproductive behavior and function. Progesterone and its metabolites, in particular, have hypnotic effects that are thought to occur via direct action on the GABA receptor complex. Unlike progesterone, estrogen appears to have the opposite effect on the GABA system in the central nervous system. Estrogen has been shown to suppress GABAmediated inhibition in the hippocampus, and have excitatory effects on the cerebral cortex and cerebellum A. Estrogens also potentiate the binding of glutamate to $\mathrm{N}$-methyl D-aspartate receptors ${ }^{[8]}$ and increase the rate of degradation of monoamine oxidase and intraneuronal serotonin transport, both of which serve to increase serotonin availability in the synapse, and therefore enhances mood ${ }^{[9]}$.

Estrogen is also a potent antioxidant and antiinflammatory agent and fluctuation in estrogen levels is encountered in various phases and interventions related to a woman's reproductive life, and periods of low estrogen are associated with mood disturbances, including depression in many women. Furthermore, changes in plasma estrogen levels are accompanied by changes in a variety of other neurotransmitters including acetylcholine, dopamine, serotonin (5hydroxytryptamine, 5-HT) and endorphins ${ }^{[10]}$.

Both dopamine and serotonin, important moodstabilizing neurotransmitters, decrease with age in both sexes ${ }^{[11]}$. Dopamine is an essential neurotransmitter and a neurohormone released by the hypothalamus. Dopamine has many functions in the brain, including important roles in behavior and cognition, motor activity, motivation and reward, inhibition of prolactin production (involved in lactation), sleep, mood, attention, and learning ${ }^{[12]}$. Serotonin acts both as a neurotransmitter and a neuromodulator ${ }^{[13]}$ and has a pivotal role in aggression, body temperature, sleep, mood, sexuality, appetite, and vomiting. Low levels of serotonin and/or dysfunctions of CNS serotonin metabolism are associated with several psychiatric (depression, schizophrenia, anxiety) disorders and neurologic (migraine, epilepsy, chronic pain) diseases [14].

The relation between general anesthesia and women's mood is essential to the optimal care of women, especially women who are experiencing disorders of mood or who are at risk for mood disorders. Accordingly, this study was designed to investigate the effect of general anesthesia on main female sex hormones; estrogen and progesterone, and biogenic amine neuromediators; dopamine and serotonin and elucidate the relationship between general anesthesia and mood through these tested parameters.

\section{Subjects and Methods}

\section{Study design and sample collection}

After ethics committee's approval and patients' informed consent (in Demerdash hospital) the study was carried out on fifty female patients aged between 25 - 40 years scheduled to undergo laparoscopic cholecystectomy with general anesthesia, which was expected to last from 1 to 2 hours. Full medical history was taken with special attention to any associated medical problems and patients were excluded if they had any psychiatric, neurological or endocrine disorders or postmenopausal state.

General anesthesia was maintained using volatile anesthesia (isoflurane with MAC of 1.2). Nondepolarizing neuromuscular blocking agents with neostigmine and atropine were administrated intravenously to get the desired effects of anesthetic agent with minimal side effects.

Patient recovery was monitored continuously after termination of anesthesia. Venous blood samples were taken from patients before and after anesthesia to compare the levels of female sex hormones (estrogen and progesterone) and neurotransmitters (dopamine and serotonin).

Blood samples were collected in 2-mL vacutainers containing EDTA as anticoagulant, and after a $2 \mathrm{~h}$ storage at $4^{\circ} \mathrm{C}$, the supernatant (plasma) was transferred to Eppendorf tubes and centrifuged at $10,000 \times \mathrm{g}$ for 10 $\min$ at $4^{\circ} \mathrm{C}$ in an Eppendorf centrifuge (A. Hettich, Tuttlingen, Germany). The obtained supernatant was stored at $-80^{\circ} \mathrm{C}$ awaiting analysis.

\section{Quantitative determination of plasma} neurotransmitters by HPLC

A high-performance liquid-chromatographic (HPLC) (Zorbax 300 SB C18 column $4.5 \times 250 \mathrm{~mm}$ ) (Agilent 1100 technologies, USA) method for measuring dopamine and serotonin in plasma was carried out as previously described by Davis et. al. ${ }^{[15]}$. These amines are modified by pre-column derivatization with o-phthalaldehyde, which stabilizes the molecules, facilitates extraction, and improves detection of nanogram amounts. Before separation, samples were neutralized with $\mathrm{KOH}$ and immediately derivatized and extracted into ethyl acetate, in which derivatives were stable for longer than $24 \mathrm{hs}$. Interfering amino acids were removed from ethyl acetate by partitioning twice with $\mathrm{Na}_{2} \mathrm{HPO}_{4}$ buffer $(\mathrm{pH} 10.0$ ) . Separation was complete in about $90 \mathrm{~min}$ on a $4.5 \mathrm{X}$ $250 \mathrm{~mm}$ C18 column", with a flow rate of $1.5 \mathrm{ml} / \mathrm{min}$ using a stepwise gradient of sodium phosphate buffer (pH 3.2). The derivatives were quantitated by measuring their fluorescence intensity (340-nm excitation, 480-nm emission).The relative weight response (RWR) values for each of the biogenic amines were determined by at least five independent analyses of calibration standards of the biogenic amines; thereafter, the RWR was determined daily.

\section{Quantitative determination of estrogen and progesterone levels}

Estrogen is a term used to include 3 major hormones (estradiol, estriol, and estrone). Estradiol is the most potent estrogen and the predominant form circulating in the body from menarche to menopause, so that measure- 
ment of plasma estradiol level will express the level of plasma estrogen.

Plasma estrogen (estradiol) and progestrone were measured by Radioimmunoassay (RIA) using commercially available kits as described previously by Saraqueta et. al. ${ }^{[16]}$. Estradiol was measured by a competitive immunoenzymatic assay (Immuno 1, Bayer,Tarrytown, NY, USA). The sensitivity of the assay was $10 \mathrm{pg} / \mathrm{ml}$ and the interassay coefficient of variation was 5\%. Progesterone was determined by a competitive chemiluminiscent immunoassay (Immulite, DPC, Los Angeles, CA, USA). The sensitivity of the method was $0.2 \mathrm{ng} / \mathrm{ml}$ and the interassay coefficient of variation was $6.7 \%$.

\section{Statistical analysis}

Data were analyzed using statistical program for social science (SPSS) version 18.0. Quantitative data were expressed as mean \pm standard deviation (SD).Statistical significance was tested among and between groups using the one-way analysis of variance (ANOVA) test.

\section{Results:}

In order to investigate the general anesthesia's effect on mood and depression in pre- and post-operative female patients, the plasma levels of female sex hormones; estrogen and progesterone, and biogenic amine neuromediators; dopamine and serotonin (5hydroxytryptamine, 5-HT) were investigated. Selection of these hormones and neurotransmitters was based on their being mood stabilizers and natural antidepressant.

\section{Neurotransmitter levels}

As shown in Fig. 1, dopamine levels were significantly increased $(\mathrm{p}<0.001)$ among post-anesthesia patients relative to the pre-anesthesia ones. These results indicate that anesthesia has a very enhancing effect on mood by increasing the most natural antidepressant, dopamine (the precursor for endogenous morphine), which helps in relieving pain even after recovering from anesthesia. In contrast, a non-significant change in the serotonin levels of post-anesthesia patients was observed as compared to the pre-anesthesia ones (Fig. 2).

\section{Progesterone and estrogen Levels}

As shown in Fig. 3, data from post-operative female patients demonstrated a significant increase $(\mathrm{P}<0.001)$ in progesterone levels (45\%) as compared to the preoperative ones. On the other side, a significant decrease in the estradiol level $(18 \%)$, as a representative of estrogen, of post-operative female patients was observed when compared with the pre-anesthesia ones (Fig.4).

\section{Discussion}

Depression is more common in females than males, where women tend to report more depressive and dysphoric symptoms during times of large hormonal changes, including menopause, premenstrual period and postpartum which suggest that hormonal changes are playing a role in these symptoms ${ }^{[17]}$.

The current findings indicate that general anesthesia doesn't cause any sort of depression or negative mood, as they induced an increase in progesterone and dopamine levels concomitant with a non significant change in serotonin levels of female patients, postoperatively, compared to the preoperative levels. In contrast, a significant decrease in estrogen levels was observed.

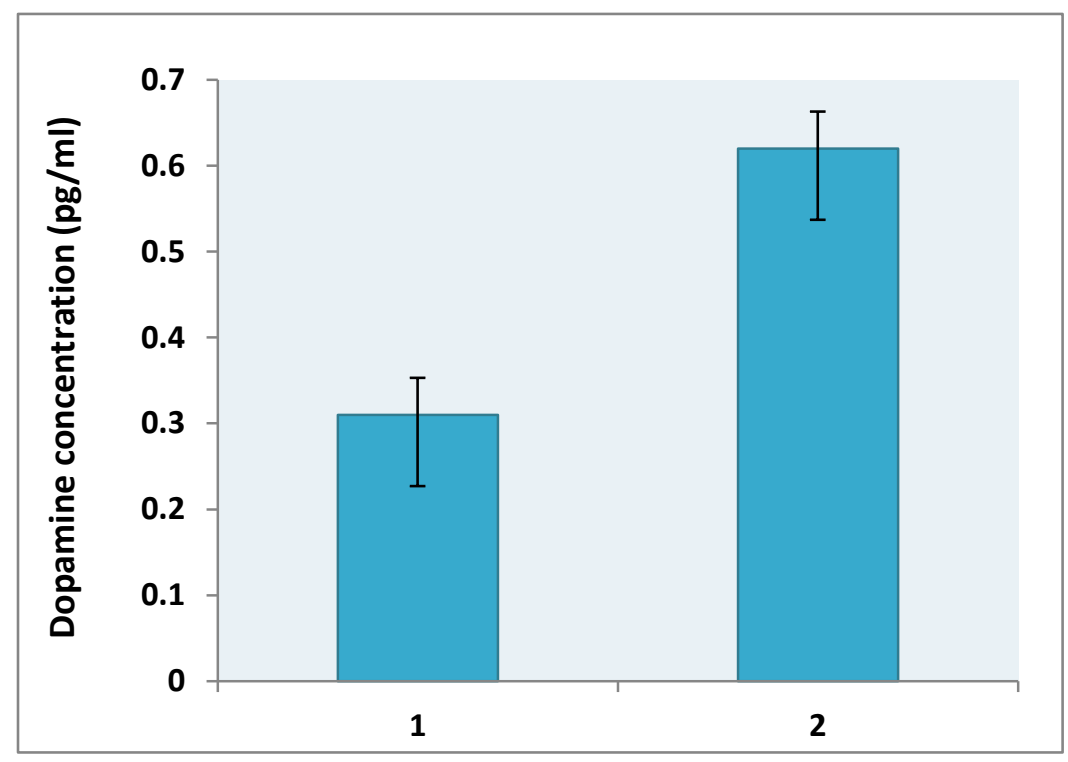

Fig. 1: Dopamine levels in pre- and post-anesthesia treated patients. 1, pre-anesthesia patients; 2, post-anesthesia patients. Values are presented as mean \pm SE for 50 patients. 


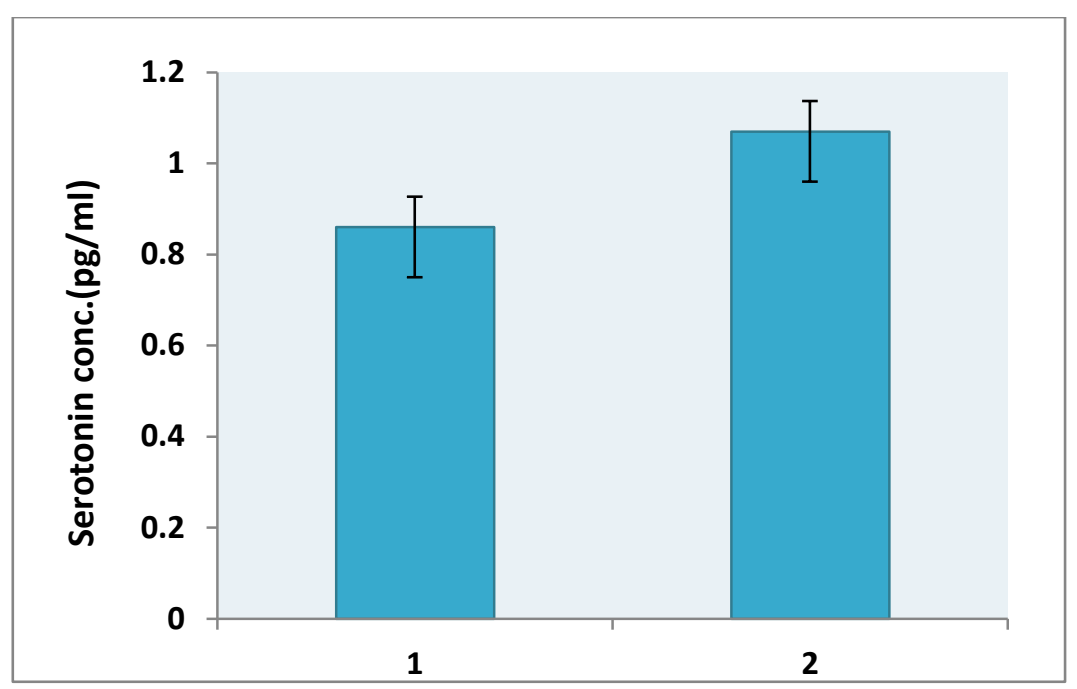

Fig. 2: Serotonin levels in pre- and post-anesthesia treated patients. 1, pre-anesthesia patients; 2, post-anesthesia patients. Values are presented as mean \pm SE for 50 patients.

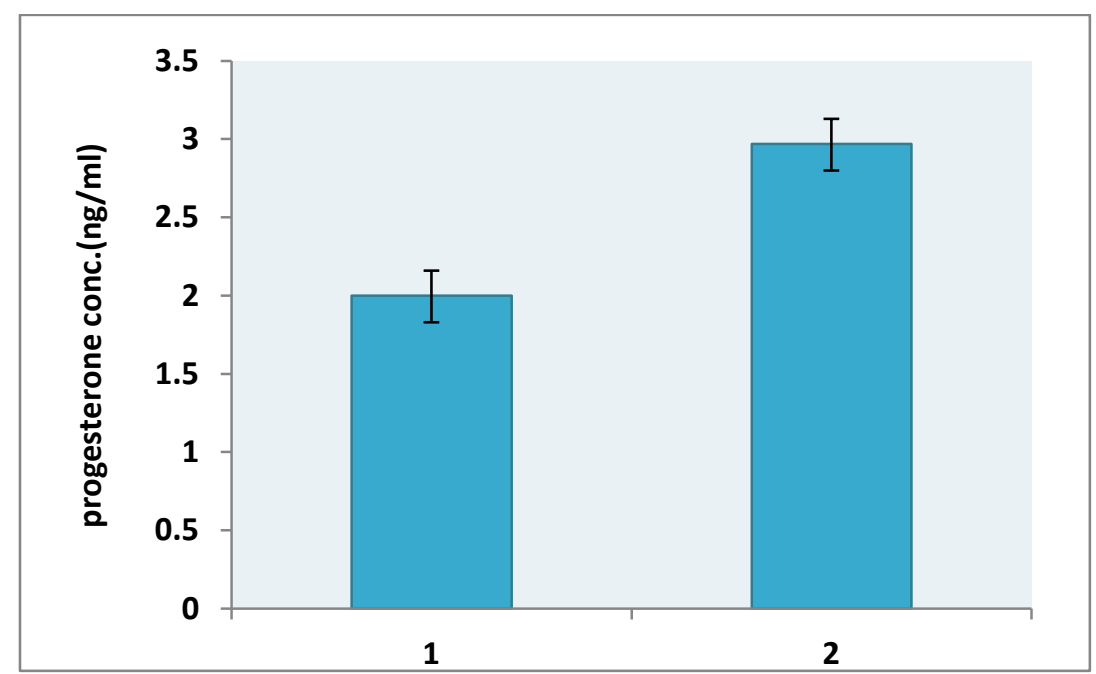

Fig. 3: Progestrone levels in pre- and post-anesthesia treated patients. 1, pre-anesthesia patients; 2, post-anesthesia patients. Values are presented as mean \pm SE for 50 patients.

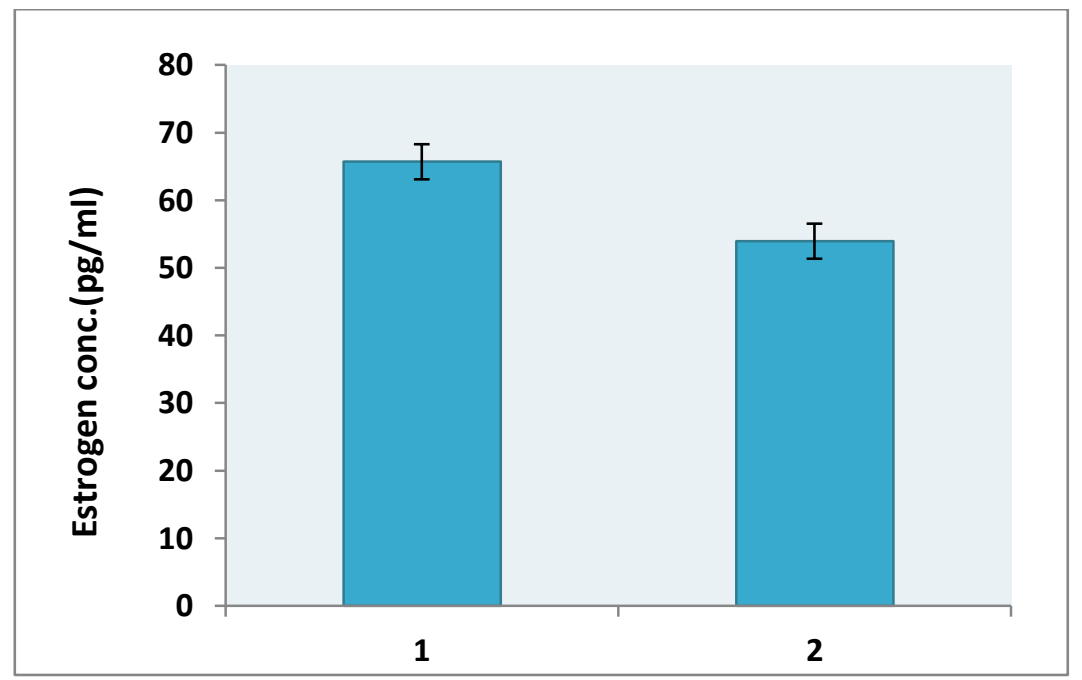

Figure (4): Estrogen levels in pre- and post-anesthesia treated patients. 1, pre-anesthesia patients; 2, post-anesthesia patients. Values are presented as mean \pm SE for 50 patients. 
Estrogen has been suggested to affect stress responsiveness and thereby is considered as a potential effect modifier of the stress response ${ }^{[18]}$. Life-style factors such as physical activity, alcohol consumption and body mass index are known to affect estrogen levels as well as morbidity and mortality ${ }^{[19]}$.

It is generally known that stressful life events are among the most potent factors that can trigger depressive episodes ${ }^{[20]}$. The stress response is mediated by the hypothalamo-pituitary-adrenal (HPA) system. Activity of the corticotropin-releasing hormone $(\mathrm{CRH})$ neurons in the hypothalamic paraventricular nucleus (PVN) forms the basis of the activity of the HPA-axis. The CRH neurons induce adrenocorticotropin (ACTH) release from the pituitary, which subsequently causes cortisol release from the adrenal cortex, which may eventually lead to an impairment of estrogen synthesis ${ }^{[21]}$. In the present study, the significant decrease in estrogen level postoperatively is in agreement with the previous findings.

It was reported that estrogen replacement therapy (ERT) may make women with Alzheimer's disease (AD) less vulnerable to depression ${ }^{[22]}$ and may augment the fluoxetine response in elderly depressed patients ${ }^{[23]}$. However, it should be noted that estrogen substitution in postmenopausal women with depressive symptoms was effective in some studies but not in others ${ }^{[24]}$. Therefore, these findings might indicate that the observed decrease in the level of estrogen postoperatively, was not an indication of depression.

The serotonin neural system regulates numerous autonomic functions including pituitary hormone secretion, satiety, or sexual behavior and serotonin contributes to cognition and affects most or all of which are sensitive to estradiol as well ${ }^{[25]}$. Many, if not all of these physiological endpoints are altered by estradiol. It was reported that estradiol induced progestin receptors (PR) in the serotonin neurons of the macaque dorsal raphe ${ }^{[26]}$. This action has been ubiquitously attributed to an action of estradiol through a nuclear receptor ${ }^{[27,28]}$. Estradiol also increased gene and protein expression for tryptophan hydroxylase (TPH), the committal enzyme in serotonin synthesis in macaques [29] which could increase serotonin neurotransmission. Therefore, the serotonin neural system may play a critical role in transducing the action of estradiol on mood as well as aspects of cognition, learning and memory ${ }^{[30]}$. Low levels of estrogen, and therefore, of brain neurotransmitters, such as serotonin, may induce depression. This may be reversed by antidepressants, such as selective serotonin reuptake inhibitors (which increase levels of neurotransmitters in the synapse) and monoamine oxidase inhibitors (which prevent the breakdown of neurotransmitters). In contrast to estrogen, progesterone increases the amount of monoamine oxidase, thereby decreasing the concentration of brain serotonin ${ }^{[31]}$.

Martin et. al. ${ }^{[32]}$ demonstrated that isoflurane, at clini- cally relevant concentrations, produced a concentrationdependent decrease in serotonin uptake, which was kinetically observed to occur in a noncompetitive manner. Based on these findings isoflurane-induced decrease in synaptosomal reuptake of serotonin would have been expected to produce an increase in extracellular serotonin; however, we observed a nonsignificant change in extracellular serotonin after isoflurane administration. Thus, we could suggest that this may have been attributable to the isoflurane inhibition of serotonin uptake, which masks the decrease in the synthesis of serotonin due to the low estrogen level and increased level of progestrone.

Dopamine (DA) is a monoamine neurotransmitter in the human brain that is involved in several physiological processes. It is also the precursor for endogenous morphine, which is the most used compound among narcotic analgesics ${ }^{[33]}$. Estrogen and progesterone have been found to affect dopaminergic function, where they enhance striatal dopamine release. Progesterone can rapidly increase dopamine release and protect dopaminergic neurons against degeneration ${ }^{[34]}$.

In the present study, there was a significant increase in progesterone and dopamine levels postoperatively. These results are in agreement with results from a previous study that reported an increase in extracellular dopamine concentration during volatile isoflurane anesthetic administration. This increase was attributed to the anesthetic effects on the dopamine transporter which is a key regulator of synaptic dopamine concentration and may mediate the increase in extracellular dopamine during anesthesia ${ }^{[3]}$.

In conclusion, these results indicate that general anesthesia using isoflurane doesn't induce negative mood or any sort of depression. Instead, it enhances mood via an induced increase in the serum levels of dopamine and progesterone and masks the decreased synthesis of serotonin (induced by decreased estrogen level) through reducing or inhibiting serotonin uptake, postoperatively. In addition, anesthetic agents help in great relieving of pain by increasing endogenous morphine through rising dopamine level (the precursor for endogenous morphine), which act as natural pain killer.

\section{References}

1) Muller, C. P., Pum, M. E., Amato, D., Schuttler, J., Huston, J. P. and Silva, M. A. (2011). The in vivo neurochemistry of the brain during general anesthesia. Journal of neurochemistry; 119(3):419446.

2) Jenkins, K. and Baker, A. B. (2003). Consent and anesthetic risk. Journal of Anesthesia; 58(10):962984.

3) Votaw, J. R., Byas-Smith, M. G., Voll, R., Halkar, R. and Goodman, M. M. (2004). Isoflurane alters the amount of dopamine transporter expressed on the plasma membrane in humans. Journal of Anesthesiology; 101(5):1128-1135. 
4) Burns, D. D. (1999). Feeling Good: The New Mood Therapy. New York: Avon Books, pp 42-43.

5) Griffiths, R., Greiff, J. M., Boyle, E., Rowbotham, D. J. and Norman, R. I. (1994). Volatile anesthetic agents inhibit choline uptake into rat synaptosomes. Journal of Anesthesiology; 81(4):953-958.

6) Fillingim, R. B. (2000). Sex, gender and pain : women and men are really different. Journal of current review of pain; 4(1):24-30.

7) Buchanan, F. F., Myles, P. S. and Cicuttini, F. (2009). Patient sex and its influence on general anesthesia. Journal of Anesthesia and intensive care; 37(2):207-218.

8) Buchanan, F. F., Myles, P. S. and Cicuttini, F. (2011). Effect of patient sex on general anaesthesia and recovery. Br. J. Anaesth; 106(6):832-839.

9) Carretti, N., Florio, P., Bertolin, A., Costa, C. V. L., Allegri, G. and Zilli, G. (2005). Serum fluctuations of total and free tryptophan levels during the menstrual cycle are related to gonadotrophins and reflect brain serotonin utilizaton. Hum. Reprod.; 20(6):1548-1553.

10) Sherwin, B. B. (1997). Estrogenic effects on the central nervous system: clinical aspects. In: Lindsay $\mathrm{R}$, Dempster DW, Jordan VC, eds. Estrogens and Antiestrogens. Philadelphia: Lippincott-Raven, pp:75-87.

11) Sherwin, B. B. (2003). Estrogen function in women. Endocr Rev.; 24:133-151.

12) Abe, K., Niikura, Y., Fujimoto, T., Akaishi, T., and Misawa, M. (2008). Involvement of dopamine D2 receptors in the induction of long-term potentiation in the basolateral amygdala-dentate gyrus pathway of anesthetized rats. Neuropharmacology; 55:1419-1424.

13) Descarries, L., Audet, M. A., Doucet, G. S., Garcia, S., Oleskevich, S., Seguela, P., Soghomonian, J. J. and Watkins, K. C. (1990). Morphology of central serotonin neurons. Brief review of quantified aspects of their distribution and ultrastructural relationships. Ann. NY Acad.Sci.; 600: 81-92.

14) Szilágyi, A., Boór, K., Orosz, I., Szántai, E., Székely, A., Kalász, H., Sasvári-Székely, M., and Farkas, V. (2006). Contribution of serotonin transporter gene polymorphisms to pediatric migraine. Headache; 46(3): 478-485.

15) Davis, T. P., Gehrke, C. W., Gehrke, C. W., Cunningham, T. D., Kuo, K. C., Gerhardt, K. O., Johnson, H. D. and Williams, C. H. (1978). HighPerformance Liquid-ChromatographicSeparation and Fluorescence Measurement of Biogenic Amines in Plasma, Urine, and Tissue Clin.Chem.; 24:13171324.

16) Saraqueta, P., Krimer, A. R. D., Charreau, E. H. and Tesone, M. (1989). Insulin regulation of steroidogenic activity in rat culture luteal cells. J.
Steroid Biochem.; 32:393-397.

17) Benmansour, S., Weaver, R. S., Barton, A. K., Adeniji, O. S., Frazer, A. (2012). Comparison of the effects of estradiol and progesterone on serotonergic function. Biol. Psychiatry.; 71:633-641.

18) Kajantie, E. and Phillips, D. I. (2006). The effects of sex and hormonal status on the physiological response to acute psychosocial stress. Psychoneuroendocrinology; 31:151-178.

19) Rod, N. H., Hansen, A. M., Nielsen, J., Schnohr, P. and Gronbaek, M. (2009). Low-risk factor profile, estrogen levels, and breast cancer risk among postmenopausal women. Int. J. Cancer; 124:19351940.

20) Kendler, K. S., Karkowski, L. M. and Prescott, C. A. (1999). Causal relationship between stressful life events and the onset of major depression. Am. J. Psychiatry; 156: 837-841.

21) Breen, K. M. and Karsch, F. J. (2004). Does cortisol inhibit pulsatile luteinizing hormone secretion at the hypothalamic or pituitary level? Endocrinology; 145:692-698.

22) Carlson, L. E., Sherwin, B. B. and Chertkow, H. M. (2000). Relationships between mood and estradiol (E2) levels in Alzheimer's disease (AD) patients. J. Geront., Ser. B Psychol. Sci. Soc. Sci.; 55: 47-53.

23) Schneider, L. S., Small, G. W., Hamilton, S. H., Bystritsky, A., Nemeroff, C. B. and Meyers, B. S. (1997). Estrogen replacement and response to fluoxetine in a multicenter geriatric depression trial. Fluoxetine Collaborative Study Group. Am. J. Geriatr. Psychiatry; 5: 97-106.

24) Rubinow, D. R., Schmidt, P. J. and Roca, C. A. (1998). a. Estrogen-serotonin interactions: implications for affective regulation. Biol. Psychiatry; 44:839-850.

25) Jacobs, B. L. and Azmitia, E. C. (1992). Structure and function of the brain serotonin system, Physiolog. Rev.; 72: 165-231.

26) Bethea, C. L. (1994). Regulation of progestin receptors in raphe neurons of steroid-treated monkeys, Neuroendocrinology; 60: 50-61.

27) Cho, H., Aronica, S. M. and Katzenellenbogen, B. S. (1994). Regulation of progesterone receptor gene expression in MCF-7 breast cancer cells: a comparison of the effects of cyclic AMP, estradiol, IGF-1 and serum factors, Endocrinology; 134: 658664.

28) Katzenellenbogen, J. A., O'Malley, B. W. and Katzenellenbogen, B. S. (1996). Tripartite steroid hormone receptor pharmacology: interaction with multiple effector sites as a basis for the cell- and promoter-specific action of these hormones, Mol. Endocrinol.; 10: 119-131.

29) Pecins-Thompson, M., Brown, N. A., Kohama, S. C. and Bethea, L. (1996). Ovarian steroid regulation of tryptophan hydroxylase mRNA expression in rhesus macaques, J. Neurosci.; 16:7021-7029. 
30) Gundlah, C., Lu, N. Z., Mirkes, S. J. and Bethea, C. L. (2001). Estrogen receptor beta (ERbeta) mRNA and protein in serotonin neurons of macaques. Brain Res Mol Brain Res.; 91(1-2):1422.

31) Douma, S. L., Barwin, B. N. and Woodend, A. K. (2005). Estrogen-related Mood Disorders Reproductive Life Cycle Factors. Advances in Nursing Science; 28: 364-375.

32) Martin, D. C., Adams, R. J. and Aronstam, R. S. (1990). The influence of isoflurane on the synaptic activity of 5-hydroxytryptamine. Neurochem Res.; 15: 969-973.
33) Neri, C., Ghelardini, C., Sotak, B., Palmiter, R. D., Guarna, M., Stefano, G. and Bianchi, E. (2008). Dopamine is necessary to endogenous morphine formation in mammalian brain in vivo. Journal of neurochemistry; 106(6):2337-2344.

34) Nestor, D., Salazar, P., Arraiza, C. G. and Velasco, I. (2007). Changes in the content of estrogen and progesterone receptors during differentiation of mouse embryonic stem cells to dopamine neurons. Journal of brain research Bulletin; 73(1-3):75-80. 